\title{
Building Pb Nanomesas with Atomic-Layer Precision
}

\author{
C.-S. Jiang, ${ }^{1}$ S.-C. Li ${ }^{2}$ H.-B. Yu, ${ }^{1}$ D. Eom, ${ }^{1}$ X.-D. Wang, ${ }^{1}$ Ph. Ebert,${ }^{3}$ J.-F. Jia, ${ }^{2}$ Q.-K. Xue, ${ }^{2}$ and C.-K. Shih ${ }^{1, *}$ \\ ${ }^{1}$ Department of Physics, The University of Texas, Austin, Texas 78712, USA \\ ${ }^{2}$ Institute of Physics, The Chinese Academy of Sciences, Beijing 100080, China \\ ${ }^{3}$ Institut für Festkörperforschung, Forschungszentrum Jülich GmbH, 52425 Jülich, Germany
}

(Received 21 November 2003; published 12 March 2004)

\begin{abstract}
We demonstrate a novel scheme for manipulating metallic nanostructures involving a macroscopic number of atoms, yet with precise control in their local structures. The scheme entails a two-step process: (a) a triggering step using a scanning tunneling microscope, followed by (b) self-driven and self-limiting mass-transfer process. By using this scheme, we construct $\mathrm{Pb}$ nanomesas on $\mathrm{Si}(111)$ substrates whose thickness can be controlled with atomic-layer precision. The kinetic barrier for the mass transfer and the underlying mechanism behind this novel manipulation are determined.
\end{abstract}

DOI: 10.1103/PhysRevLett.92.106104

PACS numbers: 68.55.Jk, 68.35.Fx, 68.37.Ef

Scientific and technological investigations of nanoelectronic devices and their model systems require fabrications of atomic or nanometer-scale patterns on solid surfaces [1-12]. By using the scanning tunneling microscope (STM), one can manage individual atoms and molecules and build up at one's wish a tiny structure, atom by atom and molecule by molecule [1-3]. However, such an atom-by-atom construction becomes impractical when one needs to construct larger nanostructures involving $10^{4}-10^{6}$ atoms. On the other hand, self-organization growth, by which nanostructures involving a huge quantity of atoms can be fabricated [9-12], lacks the precise control in local structures. Here we use an STM to demonstrate a new type of nanostructure manipulation where we engineer nanostructures involving a macroscopic number of atoms $\left(10^{5}-10^{6}\right)$, yet with precise control in their local structure.

The material systems under investigation are $\mathrm{Pb}$ nanomesas grown on $\mathrm{Si}(111)-(7 \times 7)$ surfaces. The growth of $\mathrm{Pb}$ mesas on $\mathrm{Si}(111)$ is achieved by depositing $\mathrm{Pb}$ onto atomically clean $\mathrm{Si}(111)-(7 \times 7)$ surfaces. The $\mathrm{Pb}$ atoms first form a wetting layer followed by three-dimensional mesas on the wetting layer $[13,14]$. An example of such a mesa is shown in Fig. 1(a), which has a thickness of $15 \mathrm{ML}$ (monolayer) on one side and $13 \mathrm{ML}$ on the other side due to substrate steps below. The flat-top structure is the consequence of a low surface energy for $\mathrm{Pb}(111)$ surfaces. Nevertheless, one also observes small step structures with step heights of $\sim 0.3 \AA$, reflecting a small mismatch in the layer thickness between $\mathrm{Si}(111)$ and $\mathrm{Pb}$ (111) [see Fig. 1(a)]. On such a mesa we demonstrate the ability to manipulate the layer thickness with atomic precision even when this process involves a large number of atoms.

The manipulation is performed at room temperature. It entails a two-step process: (i) application of a triggering pulse using an STM tip, followed by (ii) self-driven atomic motion. We first position the STM tip on the edge of the mesa [the position is labeled by the cross in
Fig. 1(a)]. There we increase the tip voltage to $+3 \mathrm{~V}$ and the tunneling current to $1.5 \mathrm{nA}$ for $30 \mathrm{~s}$ (this will be referred to as the triggering pulse). Then, the voltage and the current were reset to the imaging condition $\left(V_{t}=\right.$ $2 \mathrm{~V}, I_{t}=30 \mathrm{pA}$ ), to acquire the time-lapse STM images [Figs. 1(b)-1(d)], without perturbing the sample system. The lapse time shown on the image sequence is referenced to the beginning of the triggering pulse. Directly after the triggering pulse, one finds additional $\mathrm{Pb}$ atoms forming an annular island at the edge, which continues to grow spontaneously along the edge of the mesa (with no tip present, tip withdrawn) until it forms a ring wetting the edge [Fig. 1(b)]. This growth along the edge of the mesa is so fast that in most cases the complete annular ring is already observed in the next STM image $(<2 \mathrm{~min})$. In rare cases when the island is very large, we can observe an incomplete ring after the triggering pulse [see examples shown in Figs. 1(f)-1(h)]. However, here too, within less than $2 \mathrm{~min}$ (the lapse time between consecutive STM images), the complete annular ring is formed. Note that the lateral shape of the mesa remains preserved during this process. Once the annular ring is formed, the uphill mass-transfer rate slows down by more than 1 order of magnitude and could be easily followed with frameby-frame STM images [Figs. 1(b)-1(d)]. In this second phase of the growth of a complete additional layer on top of the mesa, the remaining vacancy island in the center of the annular ring is slowly reduced until it fully disappears. In this manner we can engineer the nanoscale mesa to any desired thickness with atomic precision by repeating this process of tip triggering followed by self-driven atomic motion. As shown in Fig. 1(e), one by one, we have placed $5 \mathrm{ML}$ of $\mathrm{Pb}$ atoms on top of the mesa without disturbing the mesa's lateral shape [15]. Note that without a triggering pulse the mesa remains unchanged and no additional layer grows within the observation time of several hours.

Since the volume of the island increases after the manipulation, $\mathrm{Pb}$ atoms must come from the environment 
(a) before $t=0$

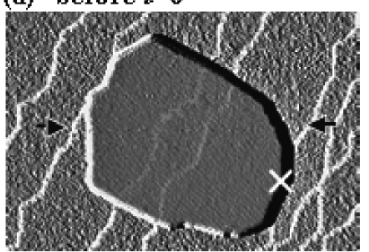

(c) $t=1440 s$
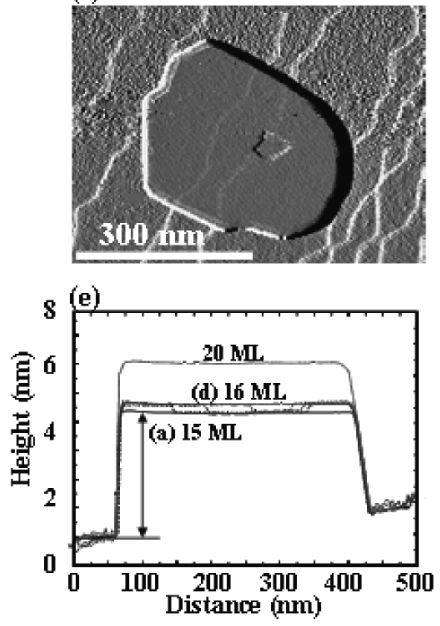

(g) $t=120 s$

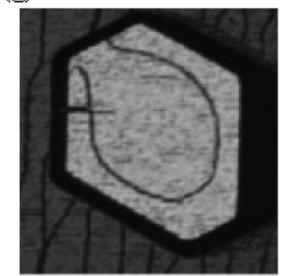

(b) $t=30 s$

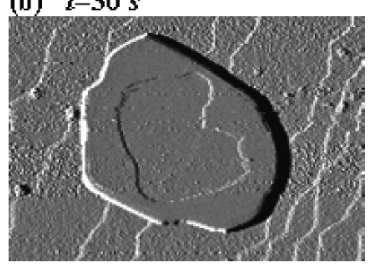

(d) $t=1740 \mathrm{~s}$

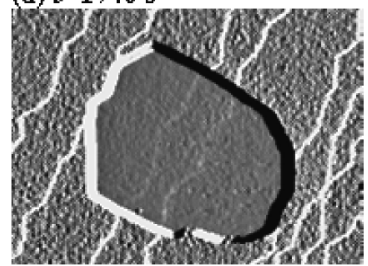

(f) $\mathrm{t}=\mathbf{0}$

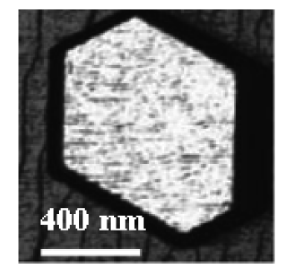

(h) $t=240 s$

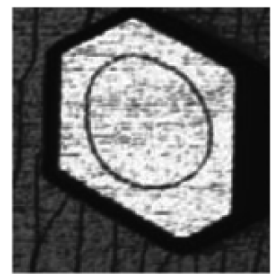

FIG. 1. Example illustrating the precise control over the thickness of a $\mathrm{Pb}$ mesa with single-layer precision. (a) shows the STM image of the mesa prior to the triggering pulse. (b) A new layer with an annular shape was triggered by positioning the tip at the edge of the mesa [white cross in (a)] at a tip voltage of $V_{y}=3 \mathrm{~V}$ and a tunnel current of $I_{t}=1.5 \mathrm{nA}$ for $30 \mathrm{~s}$. (c) Without the tip assistance, a new layer grows until the layer is completed as in (d). The profiles marked as 15 and $16 \mathrm{ML}$ in (e) correspond to the line profiles in between the arrows indicated in (a). (e) shows, in addition, a line profile (marked as $20 \mathrm{ML}$ ) across the mesa (image not shown here) obtained after repeating the manipulation process five times such that exactly $5 \mathrm{ML}$ were added on top of this mesa. (f) -(h) show a case where, after triggering, an incomplete annular shaped edge island was observed on top of a mesa. However, the edge island quickly formed closed annular shape within 2 min.

through the wetting layer. The fact that, after the triggering pulse, the mesa can grow without tip assistance indicates that thermodynamically the thicker mesa is more stable than the thinner one. The thermodynamic stability of such $\mathrm{Pb}$ mesas has recently been investigated and discussed quite intensively $[13,16,17]$. Here we focus on the kinetic issue.

The fact that without a triggering pulse a mesa with a flat (step-free) top remains unchanged shows that the

"uphill" transfer of "isolated" $\mathrm{Pb}$ atoms from the wetting layer is kinetically blocked at room temperature. The triggering pulse enables a large quantity of $\mathrm{Pb}$ atoms to be transferred to the top of the mesa (most likely a field assisted event; see below). These atoms wet the edge of the island and form a seed for the attachment of further $\mathrm{Pb}$ atoms thus effectively circumventing the kinetic barrier inhibiting at room temperature the growth of a new layer. The measurements in Fig. 1 show that this process is very fast, which slows down drastically once a complete 1 ML high annular ring wetting the mesa's edge is formed. The drastically different mass-transfer rates indicate that different mechanisms are operating during both stages of the mesa evolution:

(i) The particular growth mode characterized by the formation of an annular ring wetting the mesa's edge must involve direct jumps of $\mathrm{Pb}$ atoms from the foothill to the top and a direct attachment to the ring edge [see Fig. 2(a)]. Such an uphill mass transfer of $\mathrm{Pb}$ atoms does occur only if an island on top of the mesa is wetting the mesa's edge, indicating that individual $\mathrm{Pb}$ adatoms on the mesa's top are not able to aggregate to form a nucleation seed. This can arise only if the $\mathrm{Pb}$ adatom density on the mesa's top is too small, either because they cannot cross over the edge of the mesa or because their diffusion speed is too high, such that they effectively do not stay a long time on top of the mesa. Previous measurements during deposition of $\mathrm{Pb}$ showed that islands can nucleate on top of mesas [18], suggesting that the $\mathrm{Pb}$ adatoms cannot cross over the top edge of the mesa. This is supported by the fact that the island on top of the mesa grows preferentially along the edge, where $\mathrm{Pb}$ adatoms can move (a)

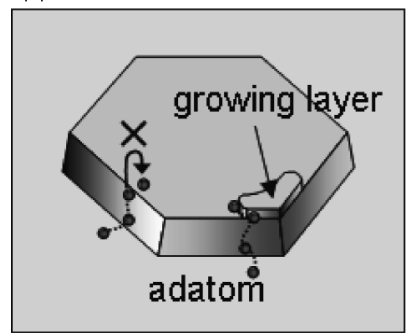

(b)

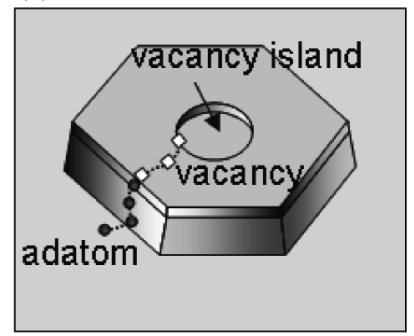

FIG. 2. Schematics showing the two different mass transport mechanisms involved. (a) illustrates the initial stage of a $1 \mathrm{ML}$ high island growing along the edge of the mesa. A direct crossover of adatoms from side walls of the nanostructure onto the top of the mesa is suppressed (marked by " $X$ ") by a large energy barrier. However, after the formation of an island at the edge of the mesa (due to the triggering pulse) the uphill mass transfer can be achieved by direct attachment of adatoms to the island at the mesa's edge without crossing of the edge, resulting in a fast growth of the island along the mesa's edge. (b) illustrates the mass transport mechanism after an annular ring is formed on top of the mesa. In this case, the mass transport is governed by the vacancy diffusion and the central vacancy island decays with time. 
uphill and be incorporated directly at the edge (no crossing of the edge is needed). The fast growth of an island along the mesa's edge also shows that $\mathrm{Pb}$ adatoms can diffuse easily on the flanks of the mesa. These flanks are in fact $\mathrm{Pb}$ surfaces with other orientations. Thus the kinetic inhibition for forming higher mesas at room temperature is due to a kinetic barrier for crossing the mesa's top edge by adatoms.

(ii) Once the annular ring is complete, such a direct attachment of $\mathrm{a} \mathrm{Pb}$ atom at the mesa's edge is no longer possible. On the other hand, as we discussed above, transferring the atoms over the edge at the top of the mesa is kinetically inhibited [see Fig. 2(a)]. Thus, the mass transfer responsible for the further growth of the new layer on the mesa must involve an alternative mechanism. As we discuss below, vacancy diffusion provides such an alternative pathway [see Fig. 2(b)]. Note that when an annular ring is formed at the edge of the mesa, equivalently there is a vacancy island at the center. Vacancies can diffuse from the edge of the vacancy island toward the edge of the mesa. Once the vacancy reaches the edge, a $\mathrm{Pb}$ atom from the wetting layer can move up to fill the vacancy site, thus completing mass transfer of one $\mathrm{Pb}$ atom to the top layer, without crossing the edge. This process continues until the top layer is completed. It then stops since no more vacancy is available.

We further investigated the detailed kinetics of this process. Figures 3(a) and 3(b) show typical data sets of the time evolution of the area $A$ and of the perimeter length $L$ of the vacancy island. Here $\Delta t=t_{o}-t_{m}$, where $t_{m}$ is the moment of measurement and $t_{o}$ is the moment when the vacancy island vanishes. The accuracy in the determination of $\Delta t$ is roughly $\pm 1 \mathrm{~min}$. As demonstrated in Fig. 3(a) the area of the vacancy island, $A$, scales with $(\Delta t)^{2}$ after the ring is closed (the slope in the log-log plot is $1.84 \pm 0.09$ ), while the perimeter of the vacancy island, $L$, is linearly dependent on $\Delta t$ (the slope in the log-log plot is $1.02 \pm 0.05)$. Consequently, one finds that the mass-transfer rate, $R(t)=-d A / d t$, is proportional to the perimeter length $L$ [Fig. 3(c)]. The mass-transfer rate per unit perimeter length is $2.1 \pm 0.3 \mathrm{~nm} / \mathrm{min}$ throughout the measurement. Different measurements performed on different mesas of very different sizes yield the same behavior; i.e., the mass-transfer rate is proportional to the peripheral length of the vacancy island, with a similar proportionality constant.

The above discussion suggests that the mass transfer is dominated by vacancy diffusion in the second phase of adding a new layer (i.e., after a complete annular ring is formed). In addition, the observed proportionality of the mass-transfer rate with the vacancy island perimeter length shows that the rate limiting process takes place at the edge of the vacancy island.

The kinetic barrier of this rate limiting process is determined using temperature dependent measurements. The resulting Arrhenius plot in Fig. 3(d) allows us to (a)

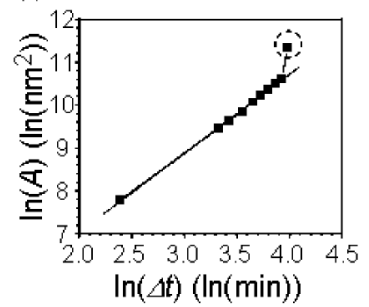

(b)

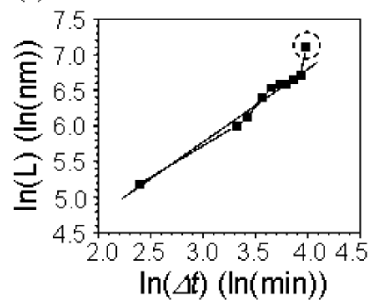

(c)

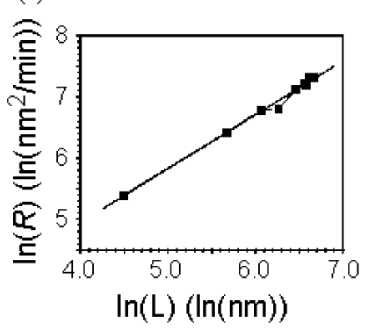

(d)

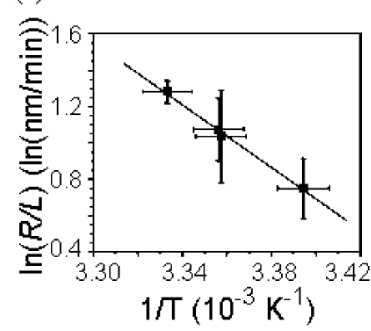

FIG. 3. Measurement of the mass transfer rate. (a) and (b) show the time evolutions of area, $A(t)$, and perimeter, $L(t)$, of the vacancy island. All data points were taken when the vacancy islands have circular shape except for those marked by circles, which were taken at the initial growth stage where the complete annular ring was not formed yet. (c) shows the mass transfer rate, $R(t)=-d A / d t$, deduced directly from the experimental data, as a function of perimeter length of the vacancy island after the annular ring was formed. (d) shows the determination of the kinetic energy barrier with normalized mass transfer rate $(d A / d t) / L(t)$ measured at different temperatures. The data also show that the mass transfer rate during the ring formation stage is about 1 order of magnitude higher. However, this could be an underestimation since in the second of the consecutive images used to calculate the rate the annular ring is already formed.

determine an activation barrier of $0.74 \pm 0.2 \mathrm{eV}$. At a closer look this activation barrier is consistent with an atomistic process. If one takes the detachment rate for individual vacancy as $K=\nu \exp (-E / k T)$, where $E$ is the activation energy, $k$ is the Boltzmann constant, and $\nu$ is an attempt frequency of typically $10^{12} \mathrm{~s}^{-1}$, then an activation energy of $0.74 \mathrm{eV}$ corresponds to a shrinking rate of $\sim 4 \mathrm{~nm} / \mathrm{min}$ for the vacancy island, very close to the experimental result of approximately $2 \mathrm{~nm} / \mathrm{min}$. The quantitative result strongly suggests that detachment of the vacancy from the vacancy island is a rate limiting process. It is unlikely that the rate limiting process is the diffusion of vacancies from the edge of vacancy island to that of the mesa, since this would reduce the effective attempt frequency and introduce a dependence of the mass-transfer rate on the width of the annular ring which is not what we have observed.

Finally, we focus on the action of the "triggering pulse." It is crucial to place the tip near the edge to effectively trigger the uphill mass transfer. Furthermore, depending on the tunneling current, there is a corresponding threshold voltage, $V_{t}$, above which the 
mass transfer can be triggered. The lower the tunneling current, the higher $V_{t}$ is. In addition, the polarity of the voltage has no discernible effect on the threshold. Since lowering the tunneling current and raising the tunneling voltage both have the effect of increasing the tip-tosample distance, it is unlikely that chemical forces at close proximity are responsible for this action [1-3]. On the other hand, the increase in the tip-to-sample distance is accompanied with the increase in $V_{t}$. It therefore strongly suggests a field-induced effect.

In summary, we demonstrated a novel nanostructure manipulation scheme that allows us to manipulate a macroscopic number of atoms with precise control of local structure. This scheme entails a two-step process, i.e., a tip triggering followed by the self-driven growth, which are associated with two different mass-transfer mechanisms: (i) direct attachment of the atoms from the foothill to the edge of the mesa during the ring formation stage and (ii) vacancy diffusion at the stage after the annular ring is completed. We further determined two rate limiting processes. (i) Without action of the tip, the crossing of the edge of the mesa on top of the mesa is inhibiting the growth of higher mesas. (ii) Once a vacancy island is formed on top of the mesa due to the growth of an annular ring, the detachment of vacancies from the vacancy island determines the mass transfer with an energy barrier of $0.74 \pm 0.2 \mathrm{eV}$.

This work was supported by the National Science Foundation (Grants No. DMR-0071893, No. DMR0306239, and No. DMR-0210383) and by Texas Advanced Research Program. S.-C. L., J.-F. J., and Q.-K.X. acknowledge the support by the NSF (Grant No. 60128404) and by MOST (2002CB613502, G001CB3095) of China.

*Corresponding author.

Electronic address: shih@physics.utexas.edu

[1] D. M. Eigler and E. K. Schweizer, Nature (London) 344, 524 (1990).
[2] M. F. Crommie, C. P. Lutz, and D. M. Eigler, Science 262, 218 (1993).

[3] H. C. Manoharan, C. P. Lutz, and D. M. Eigler, Nature (London) 403, 512 (2000).

[4] I.-W. Lyo and Ph. Avouris, Science 253, 173 (1991).

[5] L. J. Whitman, J. A. Stroscio, R. A. Dragoset, and R. J. Celotta, Science 251, 1206 (1991).

[6] C.T. Salling and M. G. Lagally, Science 265, 502 (1995).

[7] A. Kobayashi, F. Grey, R. S. Williams, and M. Aono, Science 259, 1724 (1993).

[8] T.W. Fishlock, A. Oral, R. G. Egdell, and J. B. Pethica, Nature (London) 404, 743 (2000).

[9] K. Kern et al., Phys. Rev. Lett. 67, 855 (1991).

[10] T. M. Parker, L. K. Wilson, N. G. Condon, and F. M. Leibsle, Phys. Rev. B 56, 6458 (1997).

[11] R. Notzel, Z. Niu, M. Ramsteiner, H.-P. Schonherr, A. Tranpert, L. Daweritz, and K. H. Ploog, Nature (London) 392, 56 (1998).

[12] M. Seul and D. Andelman, Science 267, 476 (1995).

[13] I. B. Altfeder, K. A. Matveev, and D. M. Chen, Phys. Rev. Lett. 78, 2815 (1997).

[14] H. H. Weitering, D. R. Heslinga, and T. Hibma, Phys. Rev. B 45, 5991 (1992).

[15] In the work of $H$. Okamoto, Dongmin Chen, and T. Yamada, Phys. Rev. Lett. 89, 256101 (2002), the STM tip was also used to perturb the $\mathrm{Pb}$ islands, however, with much stronger perturbations, such that the island shape was completely altered. They focused on the shape relaxation and the island's volume was conserved. On the other hand, the $\mathrm{Pb}$ island in this study preserves the lateral shape precisely. With the "trigger-andwait" process, we add precisely $1 \mathrm{ML}$ on top of the $\mathrm{Pb}$ mesa. Moreover, the $\mathrm{Pb}$ mesas under study have large thickness where the quantum size effect becomes less significant.

[16] Z. Zhang, Q. Niu, and C.-K. Shih, Phys. Rev. Lett. 80, 5381 (1998).

[17] I. B. Altfeder, D. M. Chen, and K. A. Matveev, Phys. Rev. Lett. 80, 4895 (1998).

[18] J. J. Métois, K. Takayanagi, and Y. Tanishiro, Surf. Sci. 155, 53 (1985). 